\title{
The Role of Internal Electric Fields in III-N Quantum Structure
}

\author{
P. Perlin, S.P. Łepkowski, H. Teisseyre, T. Suski \\ High Pressure Research Center "Unipress" \\ Sokołowska 29/37, 01-142 Warszawa, Poland
}

N. GRANdJEAN AND J. Massies

CRHEA - Centre National de la Recherche Scientifique, 06560 Valbonne, France

\begin{abstract}
Binary nitrides: of wurtzite GaN, AlN, InN, and their solid solutions represent a family of semiconductors of crucial importance for modern optoelectronics. Strained quantum wells, like GaN/AlGaN and specially InGaN/GaN, form active layers of the light emitters working in green-UV part of the spectrum. The operation of these devices strongly depends on the emission spectra of considered quantum structures which are greatly influenced by the presence of built-in electric fields. The electric field acting via quantum confined Stark effect in the mentioned structures changes the energies and intensity of the emitted light. The effect can lead to the spectral shift of a photo- and electroluminescence by many hundreds of meV. In this review we will briefly cover the influence of internal electric fields on both optical and electrical properties of nitride based heterostructures and quantum wells. We would like to draw reader's attention to the usefulness of high-pressure investigation in the study of electric fields in nitrides and to show how the interpretation of these experiments influences the way we calculate the electric fields in the quantum structures.
\end{abstract}

PACS numbers: $78.20 . \mathrm{Hp}, 78.55 . \mathrm{Cr}, 78.67 . \mathrm{Hc}$

\section{Introduction}

GaN, InN, AlN, and their solid solutions represent the newly emerged group of semiconductors, which found a number of applications in modern optoelectronics (light emitting diodes, laser diodes, visible- and solar-blind detectors) and in 
high-power, high-temperature, and microwave frequency electronics [1]. While it was known that due to omnipresence of strain (mismatch of substrates and/or different nitride alloys) all these materials should have a piezoelectric response, it was much less known that the symmetry of their wurtzite lattice allows for existence of spontaneous dielectric polarization, otherwise characteristic of ferroelectrics materials. It took quite a while until the scientific community working in the field of group III nitrides realized, in full, the importance of the dielectric polarization phenomena in nitride structures. Seen from today's perspective it may come as a surprise, but this delay had its own reason. Semiconductor physicists who, in overwhelming majority, dealt either with silicon or with GaAs and their cousins, had learned that piezoelectricity could cause, in typical structures, only tiny effects, interesting maybe as a subject for a subtle, purely academic study but irrelevant for, say, a device engineer. Extrapolation of the experience gained during the investigation of standard systems to nitrides obviously failed. Though not mysterious, the reason for this is quite a complex. The strength of polarization effect in nitrides is a result of many coinciding factors: wurtzite hexagonal symmetry of the crystal lattice allowing for the existence of spontaneous polarization, very large (one order of magnitude higher than in GaAs) piezoelectric constants, large strain present in quantum structures like GaN/AlGaN, InGaN/GaN. We should also stress the importance of the fact that almost all nitride structures are grown along (0001) polar direction of the wurtzite lattice, unlike e.g. InGaAs/GaAs structures which are grown commonly in (001) nonpolar direction of the cubic lattice [1]. In 1997 Bernardini, Fiorentini et al. [2] published the first of the series of papers on the calculation of piezoelectric constants and spontaneous polarization in nitrides which made them probably most quoted authors in this field. From this time on we can observe steadily growing number of papers devoted to the dielectric polarization phenomena in group III nitride structures. However, as we will try to show in the coming sections, full understanding of this problem has not been reached yet.

\section{Electrical polarization trivia}

Total dielectric polarization in nitrides can be expressed as a sum of piezoelectric and spontaneous components as shown in the equation below

$$
P_{\text {tot }}=P_{\mathrm{pz}}+P_{\mathrm{sp}}
$$

It is worthy noticing that the dielectric polarization leads to the appearance of fixed polarization charges and electric fields only if there are polarization gradients or discontinuities in the material. For a sufficiently narrow slab of material embedded by two semi-infinite claddings (e.g. a quantum well with thick barriers) we may write [1]:

$$
E=-\frac{\Delta P_{\text {tot }}}{\varepsilon_{\mathrm{qw}}}
$$


where $\Delta P_{\text {tot }}$ is the polarization difference between quantum well and cladding materials and $\varepsilon_{\text {qw }}$ is the static dielectric constants for quantum well. For multiquantum well structures the so-called periodic boundary conditions are usually used [1] leading to the following expressions for the electric field inside quantum wells and barriers:

$$
E_{i}=\frac{\sum_{k=\mathrm{qw}, \mathrm{br}} l_{k}\left(P_{\mathrm{tot}, k}-P_{\mathrm{tot}, i}\right) / \varepsilon_{k}}{\varepsilon_{i} \sum_{k=\mathrm{qw}, \mathrm{br}} l_{k} / \varepsilon_{k}}, \quad i=\mathrm{qw}, \mathrm{br},
$$

where $l_{k}, k=q \mathrm{w}$, br are the total thicknesses of quantum wells and barriers, respectively, $P_{\text {tot }, k}=P_{\mathrm{pz}, k}+P_{\mathrm{sp}, k}$, and $\varepsilon_{k}, k=q \mathrm{w}$, br denote the static dielectric constants for quantum well and barrier materials. In order to calculate the field we need basically only the values of the total dielectric polarization in each of the layers. Bernardini, Fiorentini et al. [2] calculated both the values of spontaneous polarization and piezoelectric constants for considered binary nitrides. Their results are gathered in Fig. 1a, b. It is worth noting, at this point, that since the dif-
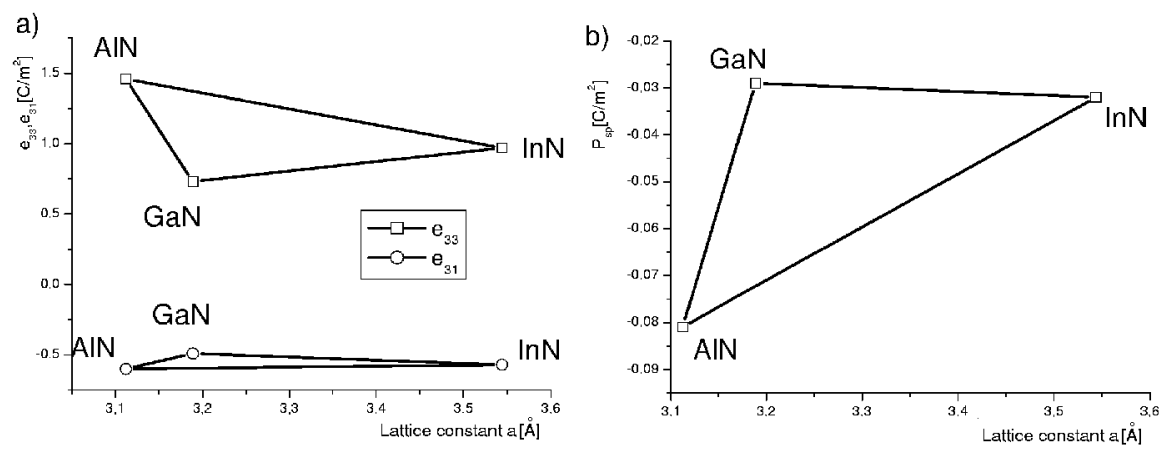

Fig. 1. Polarization parameters of binary nitrides: (a) piezoelectric tensor elements $e_{31}$ and $e_{33}$, (b) values of spontaneous polarization for three binary nitrides. Data after Ref. [2].

ferences between spontaneous polarization in $\mathrm{GaN}$ and $\mathrm{InN}$ are not large we can expect very small contribution of this spontaneous polarization in $\mathrm{GaN} / \mathrm{InGaN}$ quantum wells. In order to calculate the piezoelectric polarization in nitrides we must know the strain in each layer forming a nitride structure. Once strain is known we can get $P_{\text {piezo }}$ using a simple expression

$$
P_{\mathrm{pz}, i}=2 e_{31, i} \epsilon_{x x, i}+e_{33, i} \epsilon_{z z, i}, \quad i=\mathrm{qw}, \mathrm{br},
$$

where $e_{31, i}, e_{33, i}$ are the piezoelectric constants taken for the quantum well and barriers. This type of modeling is broadly used to calculate the electric fields in the strained quantum well systems. However so far, many groups noticed that in order to reproduce the experimental results they had to significantly modify piezoelectric parameters given by Bernardini, Fiorentini et al. [2] (see e.g. Ref. [3]). We will discuss the possible reasons for this in the last section of the present paper. 


\section{Influence of the electric field on the optical transitions in nitrides built quantum wells}

The existence of the electric field in the quantum well system leads to a bending of the conduction and valence band profiles and related localization of electron and hole gases on two opposite interfaces of the quantum well. This effect is commonly denoted as a quantum confined Stark effect (QCSE) (for one of the first reports on QCSE in nitrides see e.g. Ref. [4]). There are two basic effects associated with QCSE, i.e. the decrease in the energy of optical transitions by roughly a potential drop across the well and the decrease in the matrix element of the optical transition due to decrease in the electron and hole wave functions overlap. This effect is clearly visible in Fig. 2a, b. There are three characteristic features of QCSE
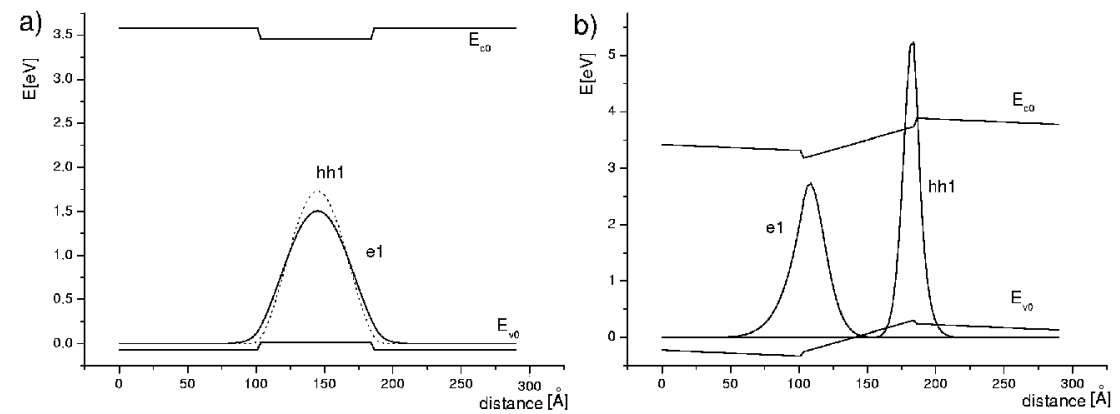

Fig. 2. Calculated wave functions for electron and holes in $\mathrm{GaN}_{\mathrm{N}} / \mathrm{Al}_{0.17} \mathrm{Ga}_{0.83} \mathrm{~N}$ quantum well ( $L_{\mathrm{qw}}=32 \mathrm{MLs}$ ): (a) no electric field, (b) with an electric field.

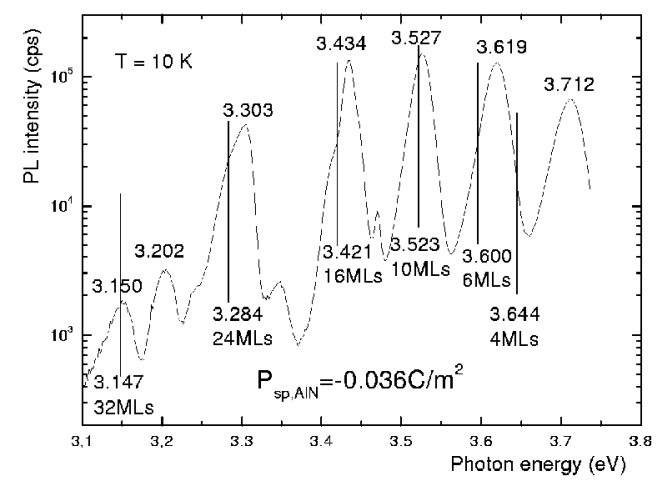

Fig. 3. Photoluminescence spectrum of a multiquantum well $\mathrm{GaN} / \mathrm{Al}_{0.17} \mathrm{Ga}_{0.83} \mathrm{~N}$ sample $(12 \mathrm{~K})$. Starting from 16 monolayers quantum well emission energy falls below that characteristic of bulk GaN. Data after Ref. [5]. 


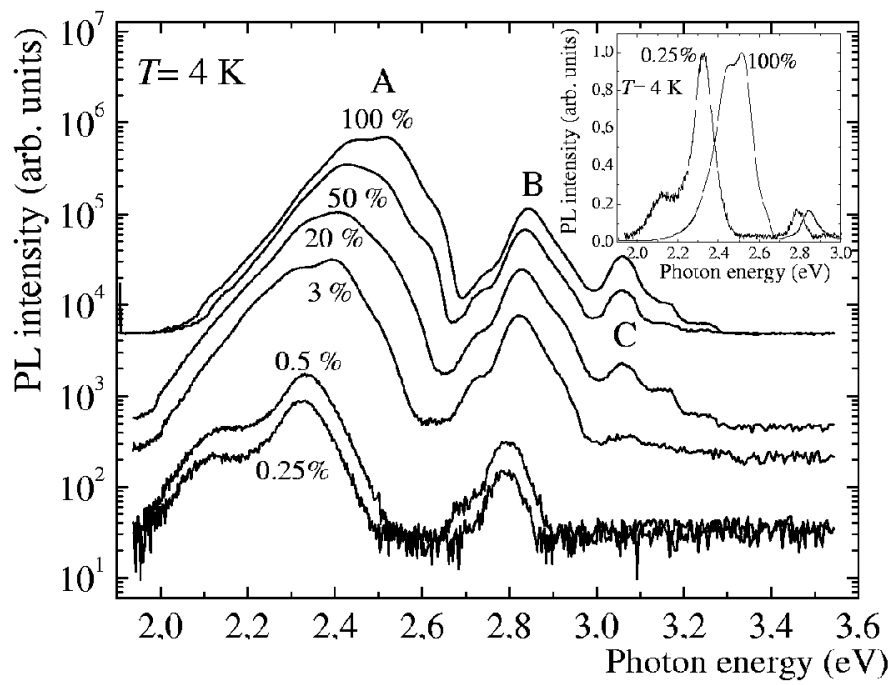

Fig. 4. Excitation power induced shift of the photoluminescence spectrum from InGaN/GaN quantum well. Inset shows similar spectra after normalization. For details see Ref. [6].

which we can use to identify this effect in quantum wells. First one is a very strong dependence of the emission energy on the quantum well thickness. We are used to the fact that in flat-band quantum structures (without electric field), the thickness of a quantum well influences the confinement energy of carriers, but in that case, with increasing well thickness, the recombination energy decreases asymptotically to the band-gap energy of the material. In the case of QCSE, with increasing well thickness, the energy drops easily much below the characteristic band-gap energy. This type of behavior is to be seen in Fig. 3, which demonstrates results obtained for the GaN/AlGaN quantum wells system [5]. The second effect is a blue shift of the emission peaks with increasing excitation power (current injection or laser intensity). The latter phenomenon originates in the screening of the electric polarization by injected or photogenerated carriers. The excitation power induced shift may easily exceed $100 \mathrm{meV}$ as it is shown in Fig. 4 for GaN/InGaN quantum wells [6]. However, even for very large pumping, full screening may not be achieved for some structures because of e.g. carrier escape from the quantum well region. The other effect resulting from electron-hole separation emerges as an increase in the emission decay time.

\section{Influence of the electrical polarization on the electrical properties of the nitrides heterostructures}

For majority of device-physicists, the most important aspect of the dielectric polarization in nitrides is the huge two-dimensional electron gas (2DEG) concen- 
trations (more than $10^{13} \mathrm{~cm}^{-3}$ ) not only in modulation doped but also in undoped $\mathrm{GaN} / \mathrm{AlGaN}$ heterostructures. This type of structures is important because of their direct application in a new generation of high electron mobility transistors (HEMT) capable of working at $\mathrm{GHz}$ frequencies and at elevated temperatures. These devices consist of semi-insulating layer of GaN with thin (200-300 $\AA$ ) layer of AlGaN. Because of the lattice mismatch between GaN and AlN (2.5\%) and the fact that AlGaN layer is deposited on the unstrained GaN layer, the thinner AlGaN layer is strained which causes the generation of piezoelectric field directed from the GaN/AlGaN interface towards the surface of the sample. This field leads to the very effective transfer of electrons from AlGaN layer to 2DEG conduction channel at GaN/AlGaN interface. Thus even without any doping (standard for GaAs/AlGaAs HEMTs) we can achieve very high 2DEG electron concentrations of the order of $10^{13} \mathrm{~cm}^{-2}[7-10]$.

Other interesting application of the dielectric polarization in the nitride structures is the control of the hole concentration in $p$-type GaN or AlGaN. This problem has special importance for a construction of special structures with improved hole concentration. Because of large ionization energy $(\approx 160 \mathrm{meV}$ ) of the commonly used $\mathrm{Mg}$ acceptor, room-temperature hole concentration is not higher than a few percents of acceptors concentration (typically lower than $5 \times 10^{19} \mathrm{~cm}^{-3}$ ). To overcome this limitation Kozodoy et al. [11, 12] proposed that in $\mathrm{Mg}$ doped short period superlattice, a strong modification of the band profiles due to piezoelectric field causes magnesium acceptor level to plunge below the Fermi level thus leading to the total ionization of all $\mathrm{Mg}$ states in the area of GaN/AlGaN interfaces and its close vicinity. Kozodoy et al. [11, 12] found that such band structure engineering can enhance the hole concentration by the factor of 10 up to $5 \times 10^{18} \mathrm{~cm}^{-3}$.

Last but not least, we have to mention a range of polarization related phenomena like pyroelectricity (manifestation of the spontaneous polarization) [13] and whole range of piezoresistive effects [14].

\section{Role of high pressures in the study of polarization effects in nitrides}

For the last few years, high pressure studies on nitride based quantum wells have attracted a considerable attention. This is because of the discovery of anomalously small pressure coefficients of the light emission in InGaN/GaN quantum wells [6, 15-17]. That discovery constituted one of many puzzling features of InGaN alloy and it was at that time attributed to localization of electrons in In-rich regions of the material, as it was originally proposed by Chichibu et al. [18]. However, recently the evidences were gathered indicating that the reduction of the pressure coefficient of the emission in InGaN quantum structures does not depend on the homogeneity in In-content distribution of the considered samples [19]. It seems that this reduction scales well with the decrease in the light emission energy in various InGaN structures. 
Explanation of this pressure anomaly can be based on two mechanisms. In the first one, we consider a peculiarity of InGaN band structure [20, 21]. This problem has been extensively investigated recently and it turned out that the pressure behavior of the alloy band structure can account only in part for the huge magnitude of the observed effect [20]. The second factor is the presence of piezoelectric fields. In order to explain surprisingly small pressure coefficients of the transition energies by the QCSE one has to assume that with the increasing hydrostatic pressure an electric field existing in the studied quantum wells increases as well. This assumption has a clear conflict with a standard understanding of the behavior of strained quantum well systems. It is a quite well-known fact that the application of the hydrostatic stress leads to the reduction of the built-in biaxial strain [22-24]. This results from the fact that materials characterized by larger lattice constants are more compressive than those of the shorter lattice constants. Thus standard linear elasticity models predicted that in InGaN/GaN quantum wells, piezoelectric field should decrease with pressure, leading to an increase and not to a decrease in the transition pressure coefficients [20].

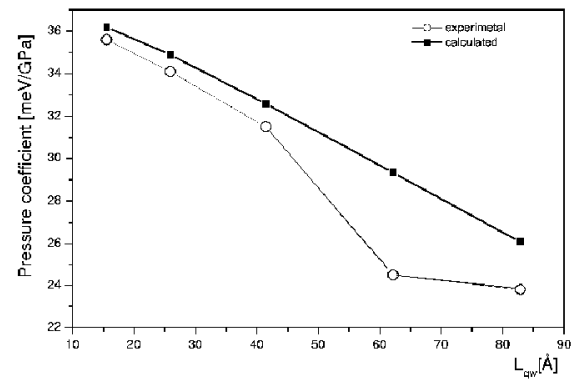

Fig. 5. Pressure coefficients of the light emission from GaN/AlGaN quantum wells as a function of the well thickness. Data after Ref. [5].

To verify the influence of hydrostatic pressure on the piezoelectric field we have chosen GaN/AlGaN quantum structure having in mind a few reasons. Firstly, light emission takes place in the binary material, i.e., in the GaN quantum well, so we can neglect all alloying effects which seem to have a significant importance for InGaN. Secondly, the elastic constants and the piezoelectric tensor are better known for the GaN-AIN system than for GaN-InN alloys. The results of this study [5] are shown in Fig. 5 demonstrating the linear dependence between the pressure coefficients and the quantum well width. Calculations based on linear elasticity and $k \bullet p$ model explain very well experimental results. At this point it is instructive to recall the reader that the compressibility of AlN and GaN are almost identical, so the before mentioned effect (of reducing biaxial strain by the hydrostatic stress) is irrelevant. It was certain for us that there was a missing element which could help us in explaining the results obtained for InGaN QWs. Recently published paper 
by Vaschenko et al. [25] has led to a kind of breakthrough. They reported on the observation of the increase in radiative recombination time in InGaN/GaN quantum wells with pressure, which they interpreted as a pressure-induced increase in piezoelectric field in these structures. Looking for the theoretical explanation for this effect (having in mind the failure of standard theories) they recalled the theoretical calculations of Shimada et al. [26] who predicted the substantial changes in the piezoelectric constants (with the volume-conserving biaxial strain always present in nitrides quantum wells). Using new values of piezoelectric constant, Vaschenko et al. [25] found the increase in piezoelectric field with the applied hydrostatic pressure which was in agreement with what was found in the experiment. This example shows how important is good analysis of high pressure experiments and that the results may be important also for good interpretation of the ambient pressure experiments. For instance, taking into account the biaxial strain dependence of the piezoelectric tensor may greatly improve the reliability of the electric fields magnitude determination in InGaN/GaN and GaN/AlGaN quantum wells based in interpretation of various experimental results.

\section{Conclusions}

We have demonstrated that the dielectric polarization in nitride based quantum structures has a large impact on their optical and electrical properties. We have also showed how high-pressure study forced us to revise the standard method of calculating electric fields in nitrides by inclusion of biaxial strain dependence of piezoelectric constants, neglected so far. Moreover, there are other factors that may improve the evaluation of the electric fields in InGaN structures. The following effects should be taken into account: a modification of (i) elastic constants of InGaN alloys with In content (non-Vegard's law dependence), (ii) elastic constants of InGaN with volume-conserving strain [27], and (iii) piezoelectric constants of InGaN alloys with In content (non-Vegard's law dependence) [28].

Summarizing the pressure results, it seems that observations of the drastic pressure coefficient drop in InGaN quantum structure favors the light emission model based on the assumption that internal electric fields play a dominant role in the radiative recombination processes. Indium segregation model is less likely to explain the considered effects. This conclusions applies also to the ambient pressure situation encouraging us to revise a standard model of radiative recombination in group III nitride alloys.

\section{Acknowledgment}

The authors (P.P., S.P.E., T.S.) want to acknowledge the financial support from the State Committee for Scientific Research, project No. 2P03B 07018. 


\section{References}

[1] H. Morkoc, Nitride Semiconductors and Devices, Springer-Verlag, Berlin 1999.

[2] F. Bernardini, V. Fiorentini, D. Vanderbilt, Phys. Rev. B 56, 10024 (1997); V. Fiorentini, F. Bernardini, F. Della Sala, A. Di Carlo, P. Lugli, Phys. Rev. $B$ 60, 8849 (1999).

[3] M. Leroux, N. Grandjean, J. Massies, B. Gil, P. Lefevre, P. Bigenwald, Phys. Rev. $B$ 60, 1496 (1999).

[4] T. Takeuchi, S. Sota, M. Katsuragawa, M. Komori, H. Takeuchi, H. Amano, I. Akasaki, Jpn. J. Appl. Phys. 2 36, L382 (1997).

[5] S.P. Łepkowski, H. Teisseyre, T. Suski, P. Perlin, N. Grandjean, J. Massies, Appl. Phys. Lett., in print.

[6] P. Perlin, C. Kisielowski, V. Iota, B.A. Weinstein, L. Mattos, N.A. Shapiro, J. Kruger, E.R. Weber, J. Yang, Appl. Phys. Lett. 73, 2778 (1998).

[7] L. Hsu, W. Walukiewicz, Appl. Phys. Lett. 73, 339 (1998).

[8] L. Hsu, W. Walukiewicz, Appl. Phys. Lett. 74, 2405 (1999).

[9] M. Asif Khan, J.W. Yang, W. Knap, E. Frayssinet, X. Hu, G. Simin, P. Prystawko, M. Leszczyński, I. Grzegory, S. Porowski, R. Gaska, M.S. Shur, B. Beaumont, M. Teisseire, G. Neu, Appl. Phys. Lett. 76, 3807 (2000).

[10] E. Frayssinet, W. Knap, P. Lorenzini, N. Grandjean, J. Massies, C. Skierbiszewski, T. Suski, I. Grzegory, S. Porowski, G. Simin, X. Hu, M. Asif Khan, M.S. Shur, R. Gaska, D. Maude, Appl. Phys. Lett. 77, 2551 (2000).

[11] P. Kozodoy, M. Hansen, S.P. DenBaars, U.K. Mishra, Appl. Phys. Lett. 74, 3681 (1999)

[12] P. Kozodoy, Y.P. Smorchkova, M. Hansen, H. Xing, S.P. DenBaars, U.K. Mishra, Appl. Phys. Lett. 75, 2444 (1999).

[13] A.D. Bykhovski, V.V. Kaminski, M.S. Shur, Q.C. Chen, M.A. Khan, in: Proc. III-Nitride, SiC and Diamond Materials for Electronic Devices. Symposium, Eds. D.K. Gaskill, C.D. Brandt, R.J. Nemanich, Mater. Res. Soc., Pittsburgh (PA) 1996 , p. 75.

[14] R. Gaska, M.S. Shur, A.D. Bykhovski, J.W. Yang, M.A. Khan, V.V. Kaminski, S.M. Soloviov, Appl. Phys. Lett. 76, 3956 (2000).

[15] P. Perlin, V. Tota-Herbei, B.A. Weinstein, P. Wiśniewski, T. Suski, P.G. Eliseev, M. Osinski, Appl. Phys. Lett. 70, 2993 (1997).

[16] W. Shan, P. Perlin, J.W. Ager III, W. Walukiewicz, E.E. Haller, M.D. McCluskey, N.M. Johnson, D.P. Bour, Appl. Phys. Lett. 73, 1613 (1998).

[17] W. Shan, J.W. Ager III, W. Walukiewicz, E.E. Haller, M.D. McCluskey, N.M. Johnson, D.P. Bour, Phys. Rev. B 58, R 10191 (1998).

[18] S. Chichibu, T. Azuhata, T. Sota, S. Nakamura, Appl. Phys. Lett. 69, 4188 (1996).

[19] P. Perlin, T. Suski, P. Wiśniewski, I. Gorczyca, S. Łepkowski, M. Hansen, S.P. DenBaars, B. Damilano, N. Grandjean, J. Massies, in: Proc. MRS Fall Meeting, Boston 2000, Eds. U.K. Mishra, M.S. Shur, C.M. Wetzel, B. Gil, K. Kishi, in press.

[20] P. Perlin, I. Gorczyca, T. Suski, P. Wiśniewski, S. Łepkowski, N.E. Christensen, A. Svane, M. Hansen, S.P. DenBaars, B. Damilano, N. Grandjean, J. Massie, Phys. Rev. $B$, in press. 
[21] L. Bellaiche, T. Mattila, L.W. Wang, S.H. Wei, A. Zunger, Appl. Phys. Lett. 74. 1842 (1999).

[22] J.A. Tuchman, I.P. Herman, Phys. Rev. B 45, 11929 (1992).

[23] D. Bertho, J.M. Jancu, C. Juanin, Phys. Rev. B 48, 2452 (1993).

[24] R.J. Thomas, M.S. Boley, H.R. Chandrasekhar, M. Chandrasekhar, C. Parks, A.K. Ramdas, J. Han, M. Kobayashi, R.L. Gunshor, Phys. Rev. B 49, 2181 (1994).

[25] G. Vaschenko, D. Patel, C.S. Menoni, S. Keller, U.K. Mischra, S.P. DenBaars, Appl. Phys. Lett. 78, 640 (2001).

[26] K. Shimada, T. Sota, K. Suzuki, H. Okumura, Jpn. J. Appl. Phys. Part 2 37, L1421 (1998).

[27] A.F. Wright, K. Leung, M. van Schilfgaarde, Appl. Phys. Lett. 78, 189 (2001).

[28] F. Bernardini, private communication. 